\title{
Identifying Fibrillization State of $A \beta$ Protein via Near-Field THz Conductance Measurement
}

\section{Chaejeong Heo $^{1,2 ;}$, Taewoo Ha ${ }^{1 ;}$, Chunjae You ${ }^{2,3}$, Thuy Huynh ${ }^{2,3}$, Hosub Lim $^{4}$, Jiwon Kim ${ }^{5}$, Mallikarjuna Reddy Kesama ${ }^{1,2}$, Jinkee Lee ${ }^{2,4}$, Teun-Teun Kim ${ }^{1 *}$,Young Hee Lee, 1,6*}

${ }^{1}$ Center for Integrated Nanostructure Physics (CINAP), Institute for Basic Science (IBS), Suwon 16419, Republic of Korea.

${ }^{2}$ Institute for Quantum Biophysics (IQB), Sungkyunkwan University, Suwon 16419, Republic of Korea.

${ }^{3}$ Department of Biophysics, Sungkyunkwan University, Suwon 16419, Republic of Korea.

${ }^{4}$ Department of Mechanical Engineering, Sungkyunkwan University, Suwon 16419, Republic of Korea.

${ }^{5}$ Department of Integrative Biotechnology, Sungkyunkwan University, Suwon 16419, Republic of Korea.

${ }^{6}$ Department of Energy Science and Department of Physics, Sungkyunkwan University, Suwon 16419, Republic of Korea

These authors contributed equally to this work.

*Corresponding authors: cjheo@skku.edu,t.kim@skku.edu, leeyoung@skku.edu 


\section{CONTENTS:}

Note 1. Optical images of the platform for the solution and dried samples

Note 2. Terahertz near-field spectrometer

Note 3. Calculations using the terahertz time-domain conductance

Note 4. Theoretical formalism of the terahertz conductance spectra and mapping

Note 5. Evaluation of the DQ value of the dried A $\beta$ proteins fitted by the modified DrudeSmith model

Note 6. DQ values of the dried and well plate samples

Note 7. A $\beta$ protein preparation and fibrillization step 
Note 1. Optical images of the platform for the solution and dried samples

(a)

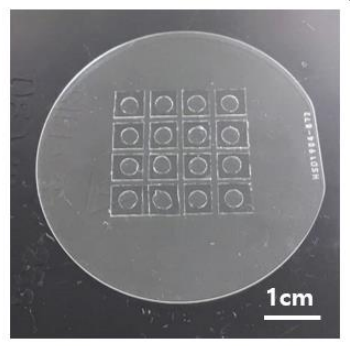

(b)

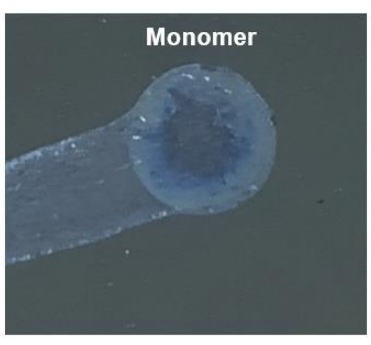

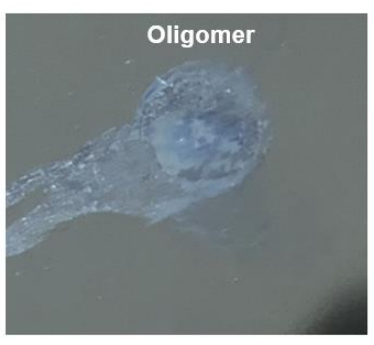

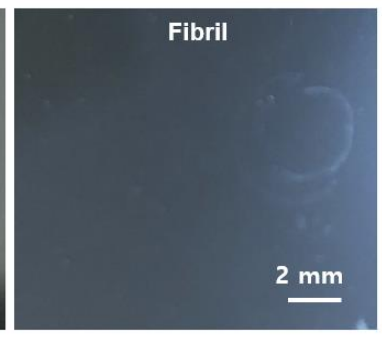

Figure S1. Optical micrograph of the sample platforms. (a) Protein sample in the buffer solution was loaded into the $(4 \mathrm{x} 4)$ PDMS well plate having a thickness of $100 \mu \mathrm{m}$ on the sapphire substrates; (b) Optical micrographs of the protein samples dropped on the $\mathrm{Si}$ wafer substrate followed by drying in air. 


\section{Note 2. THz near-field spectrometer}

Our THz near-field spectroscopy system (Figure 1b, Protemics GmbH, TeraCube Scientific) was built with a conventional THz time-domain spectroscopy (THz-TDS) system, however, we employed a unique detection method to enable the near-field imaging capability. For generating the $\mathrm{THz}$ waves, a Ti:sapphire femtosecond laser with a pulse repetition rate of $80 \mathrm{MHz}$, a pulse width of $90 \mathrm{fs}$, a center wavelength of $780 \mathrm{~nm}$ and power of $4 \mathrm{~mW}$, was used as a pump and probe source. The pump pulse was injected into the $\mathrm{THz}$ emitter to generate the $\mathrm{THz}$ waves. The probe pulse was illuminated onto a $2 \mu \mathrm{m}$ gap of Au electrode on a low-temperature grown GaAs substrate, with a $1 \mu \mathrm{m}$ thick THz tip-probe antenna (TeraSpike TD-800-X-HR-WT), and charges were subsequently induced in the GaAs substrate. Simultaneously, when the THz wave passed through the tip-probe antenna, the electric field of the $\mathrm{THz}$ wave drove the electrons to the electrodes, resulting in the flow of a current. The strength of this current was proportional to the amplitude of the THz electric field and was able to be detected using a photo-current amplifier. In this process, the dark current was observed to be below $0.4 \mathrm{nA}$ and photocurrent is above $0.1 \mu \mathrm{A}$. Conventional $\mathrm{THz}$ time-domain spectroscopy has a limitation of minimum sample size of approximately a few hundred $\mu \mathrm{m}$ due to the diffraction limit of approximately $\lambda / 2$. The tip-probe system overcomes this diffraction limit till it reaches the high spatial resolution of up to $3 \mu \mathrm{m}$. For THz mapping, highly accurate microscale-range 3D stage and measuring topography system should be needed. The stage was 3D piezo-motorized, with a $3 \mathrm{~nm}$ step resolution. The most important factor of terahertz nearfield system is the topography mapping system, because the terahertz tip is known to be very sensitive to physical external force and terahertz signal, depending on the distance between the tip and surface of the sample. For THz mapping, the distance between the tip-probe antenna and specimen can be sustained using the topography information. Therefore, the terahertz near-field spectroscopy specifies to measure the tiny size of the sample of approximately a few $\mu \mathrm{m}$ using a small gap tip-type antenna with the terahertz AC field along the $x_{-}^{-}, y_{-}$, even $z$-axis within the detection range of $0.1-2.0 \mathrm{THz}$.

The primary obstacle to $\mathrm{THz}$ spectroscopy is the attenuation by the ambient atmosphere, mainly due to intense rotational transitions of the water molecules. In gases, like water vapor, the relative isolation of the molecules results in sharp resonant peaks of the absorption centered at specific frequencies with "transmission windows" between them (Figure S2a). To avoid this unwanted 
absorption by the water vapor, dry air and nitrogen purging have been generally used. However, as shown in Figure S2b (black line), our THz near-field spectroscopy was advantageous for this significant water vapor absorption, because an optical pass length between the emitter and probe tip detector in our system was within $3 \mathrm{~cm}$. Another issue concerning water absorption is whether aqueous samples can be used for THz spectroscopy. Optical properties of water are also wellcharacterized at $\mathrm{THz}$ frequency ${ }^{1}$ and for liquid water, it was shown that the refractive index was flat, without having any absorption peaks below $1.5 \mathrm{THz}$. The red line in Figure S2b shows the transmitted $\mathrm{THz}$ signal through water. The broad transmission spectrum, without the resonance feature, can be clearly observed. However, $\mathrm{THz}$ waves were observed to only penetrate a few hundred microns in the biological specimens containing water due to the strong absorption and because of this, we carefully designed the thickness of well plate. The dynamic range is defined as signal to mean noise ratio (in Figure S2c). The maximum dynamic range of our near-field system was approximately 1700:1. Despite very small detection of below $2-3 \mu \mathrm{m}$, the value (1700:1) was found to be effective in not only measuring the near-field imaging but also in carrying out spectroscopic analysis.
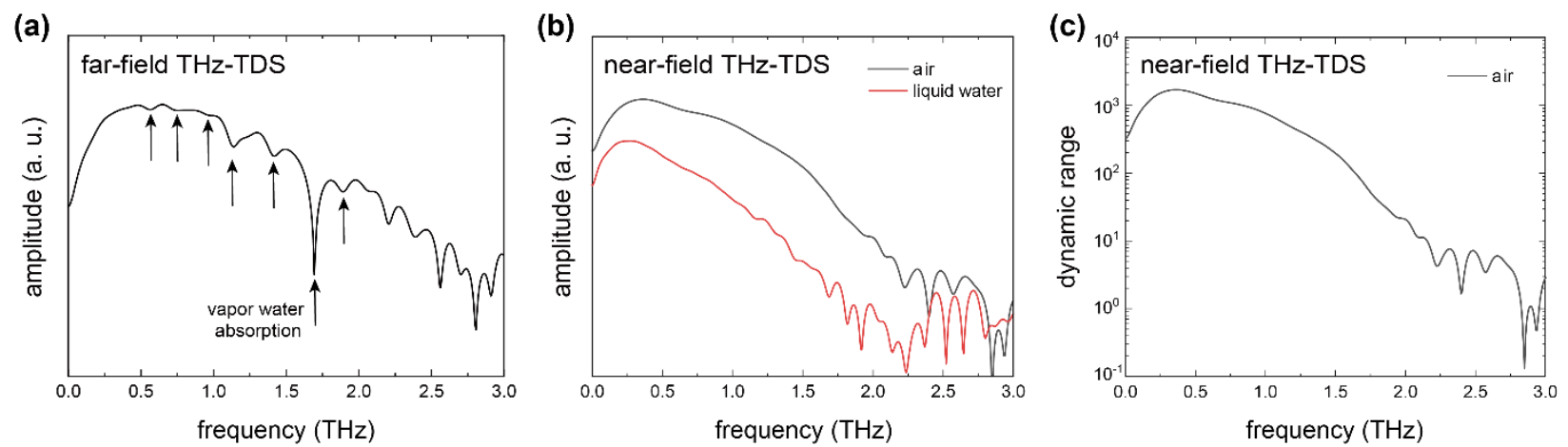

Figure S2. Comparison of the THz amplitude spectrum between (a) conventional far-field, and (b) near-field measurement system. (c) Dynamic range (amplitude/mean noise) of the THz near-field measurement system. The maximum dynamic range of our near-field system was approximately 1700:1. 


\section{Note 3. Calculations from the terahertz time-domain conductance}

The reduced intensity and phase shift were simultaneously obtained from the complex transmittance using the THz time-domain spectroscopy. Figure S3a shows the comparison of the exact and approximate refractive index of the buffer media with $100 \mu \mathrm{m}$ thickness. The difference between the exact and approximate refractive index and extinction coefficient were nearly identical over a wide range of $\mathrm{THz}(\sim 1.8 \mathrm{THz})$, except at less than $0.2 \mathrm{THz}$. In the actual experiment of measuring the $\mathrm{THz}$ conductance, the noise of the $\mathrm{THz}$ source was relatively large for less than $0.3 \mathrm{THz}$. Furthermore, the $\mathrm{THz}$ amplitude exponentially decreased after $1.1 \mathrm{THz}$. Therefore, we considered the physical conductance measurement within the THz range of $0.3-1.1$ THz for accuracy.

(a)

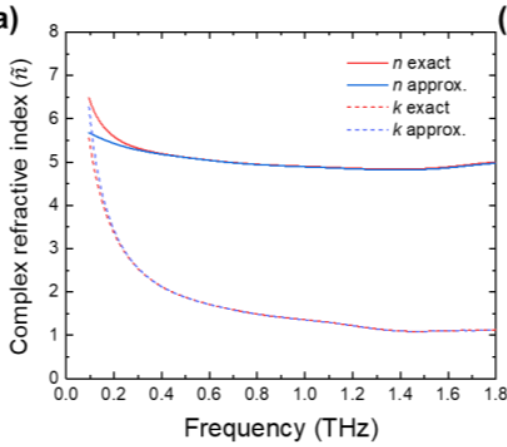

(b)

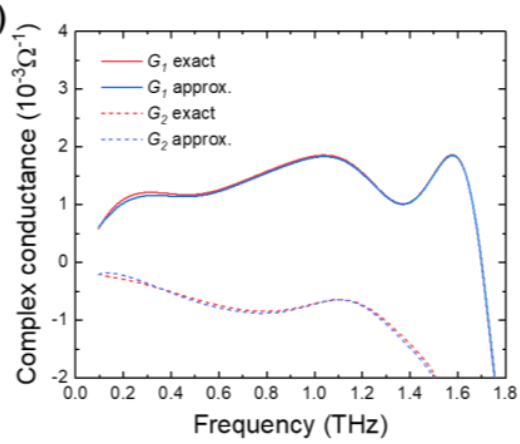

(c)

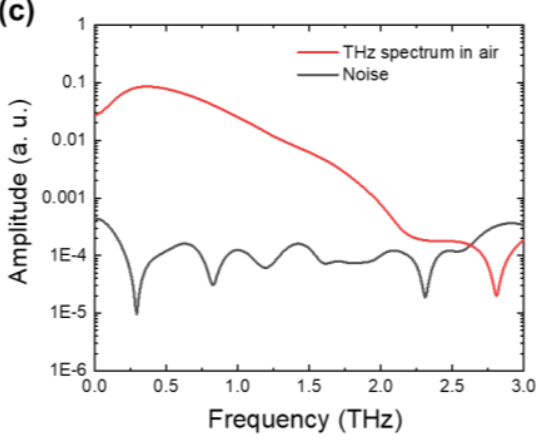

Figure S3. (a) Complex refractive index (ñ) with refractive index (n) and extinction coefficient (k) and (b) complex conductance of the buffer media with exact calculation (red line) and approximation (blue line). (c) Amplitudes of the THz spectrum and noise spectrum. 


\section{Note 4. Theoretical formalism of terahertz conductance spectra and mapping}

We performed terahertz time signal mapping at the maximum intensity of the time signal. To obtain the conductance mapping, we simplified real conductance using the conventional optical constant relation between the complex dielectric constant (and the complex conductivity):

$$
\epsilon(\omega)=\epsilon_{L}(\omega)+\frac{i \sigma(\omega)}{\omega \epsilon_{0}}
$$

where the lattice component is associated with a high frequency $\epsilon_{L}(\omega)$ or low-frequency dielectric constant $\epsilon_{\infty}, \epsilon_{s}$. From Eq. S1, the conductivity of the sample can be expressed as:

$$
\tilde{\sigma}(\omega)=\frac{2}{Z_{0} d}\left(\frac{1}{\tilde{T}(\omega)}-1\right)+i\left(\epsilon_{L}+1\right) \omega \epsilon_{0}
$$

where the impedance of free space is $Z_{0}=\sqrt{\mu_{0} / \epsilon_{0}}$. We can estimate the conductance from the complex transmittance $\tilde{T}(\omega)$ as follows:

$$
\tilde{G}(\omega)=\frac{2}{Z_{0}}\left(\frac{1}{\tilde{T}(\omega)}-1\right)+i\left(\epsilon_{L}+1\right) \omega \epsilon_{0}
$$

We considered only the real part conductance and ignored the imaginary part.

$$
G_{1}(\omega)=\frac{2}{Z_{0}}\left(\frac{1}{T(\omega)}-1\right)
$$

If the transmission level in the $\mathrm{THz}$ range did not considerably change with the frequency, we can estimate the real conductance value related to the transmission by using only the peak intensity ratio of the terahertz time signal between the media solution and $\mathrm{A} \beta$ contained media solution as follows: ${ }^{2,3}$

$$
\bar{G}_{1}=\frac{2}{Z_{0}}\left(\frac{1}{\bar{T}}-1\right)
$$

The averaged real conductance level was reasonably consistent with the real part conductance obtained via complex calculation as shown below. 
The terahertz pulse detected with time, $\mathrm{E}(\mathrm{t})$, can be transformed to a complex power spectrum in the frequency domain composed of amplitude $(\rho(\omega))$ and phase $(\phi(\omega))$ terms using the Fourier transformation.

$$
\tilde{E}(\omega) \equiv \int E_{T H z}(t) e^{-i \omega t} d t=\rho(\omega) e^{-i \phi(\omega)}
$$

Terahertz transmission is defined as the ratio of the sample to reference (in our case air) electric field as a function of frequency, $E_{s}(\omega) / E_{r}(\omega)$. The complex terahertz conductance in the sapphire/solvent/sapphire sandwich structure can be calculated using the terahertz transmission coefficient, which includes the amplitude and phase information corresponding to the real and imaginary parts of the wave equation (details in the main text).

In general, in the solid state, most specimens were formed as thin films, fully separated with a thin film and substrate. Using the Fresnel equation, we can extract the optical constant of the film. For example, in the case of conducting thin solid film, complex conductivity of the thin film can be extracted using the transmittance ratio between the thin film+substrate and substrate based on the Tinkham's formula, ${ }^{4}$ while, nothing existed in the boundary between the sample and substrate in the case of soft matter. If the volume of the $A \beta+$ buffer and buffer are the same, we can estimate the conductance of the $A \beta$ protein using the follow equations. In our system, the transport model can be represented as a parallel connection of the resistance, as follows:

$$
\frac{1}{R_{A \beta+b u f f e r}}=\frac{1}{R_{A \beta}}+\frac{1}{R_{\text {buffer }}} .
$$

where, $R_{A \beta+b u f f e r}, R_{A \beta}$ and $R_{\text {buffer }}$ are the resistance of buffer solution with $\mathrm{A} \beta, \mathrm{A} \beta$ protein and buffer solution without $\mathrm{A} \beta$, respectively.

The resistivity can be changed by the conductivity based on the relation between resistance and conductance, as follows:

$$
\tilde{G}_{A \beta+b u f f e r}=\tilde{G}_{A \beta}+\tilde{G}_{b u f f e r}
$$

where, $\tilde{G}_{A \beta+b u f f e r}, \tilde{G}_{A \beta}$ and $\tilde{G}_{b u f f e r}$ are the complex conductance of buffer solution with A $\beta$, $\mathrm{A} \beta$ protein and buffer solution without $\mathrm{A} \beta$, respectively.

The equation (S8) can be expressed as 


$$
\widetilde{G}_{A \beta}=\widetilde{G}_{A \beta+b u f f e r}-\widetilde{G}_{b u f f e r}
$$

Here, $\tilde{G}_{A \beta}$ is a complex quantity. 
Note 5. Evaluation of the DQ value of the dried A $\beta$ proteins fitted by the modified DrudeSmith model

The Drude-Smith formula is useful to describe the localized carrier behavior of a non-interacting charged particle. The Drude-Smith model is phenomenologically extended from Drude model, where a carrier partially preserves the initial velocity upon scattering. The model also describes that randomly distributed collisions in time are presumed and the fraction $c$ of the initial velocity of the electron with the scattering event allows for the Drude model to be generalized as follows:

$$
\tilde{\sigma}(\omega)=\frac{\sigma_{D C}(0)}{1-i \omega \tau}\left[1+\frac{c}{1-i \omega \tau}\right]
$$

where $\sigma_{D C}, \omega, \tau$, and $\mathrm{c}$ represent the DC conductivity, angular frequency, scattering time and localized parameter.

Localized factor implies the degree of electron backscattering. This factor can widely vary from weak to highly localized system whenever there is a localization effect of the charge. We can compare the localization parameter of each sample using the following modified Drude-Smith model:

$$
\widetilde{G}(\omega)=\frac{G(0)}{1-i \omega \tau}\left[1-\frac{D_{e}}{(1-i \omega \tau)}\right]
$$

where, $\mathrm{D}_{\mathrm{e}}$, called the dementia factor, is identified to the degree of carrier localization.

In this study, we phenomenologically estimated the De of the $\mathrm{A} \beta$ proteins with different fibrillization states in a complex solvent media. To estimate the DQ value, a modified Drude-Smith analysis was conducted with the real and imaginary conductance of the dried A $\beta$ proteins on a lowdoped silicon substrate $(\sim 100 \Omega)$. The pure silicon substrate helped in elucidating a broader terahertz conductance spectrum, due to its high transparency within the entire terahertz ranging from 0.1 to $2 \mathrm{THz}$. The extracted DQ values of the dried A $\beta$ proteins were observed to be similar to those of the solution. However, fluctuation in the complex conductivity resulted in a phase deviation due to the inhomogeneous surface of the dried specimens. Consequently, the estimated DQ values of the dried $A \beta$ proteins resulted in larger standard deviations compared to the values of the solution-based specimens. 


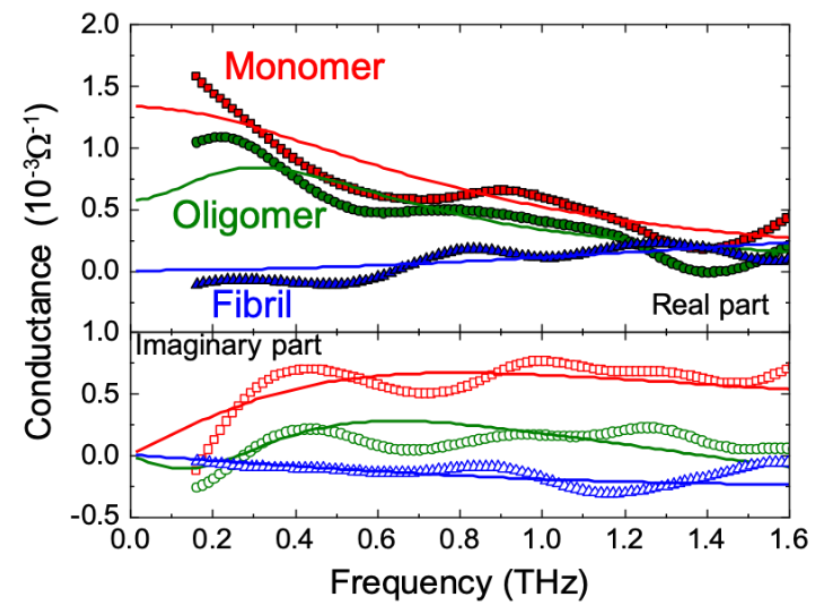

Figure S4. Real and imaginary conductances with dried monomer, oligomer, and fibril A $\beta$ proteins, on the Si wafer fitted using the modified Drude-Smith model, with experimental data. 


\section{Note 6. DQ values of the dried and well plate samples}

Accurate quantification for the fibrillization of $\mathrm{A} \beta$ proteins is one of the most important and difficult issues. Terahertz near-field spectroscopy can estimate precise DQ, regardless of the concentration of the A $\beta$ protein. Figure S6 shows the discretized DQ values of both the dried and solution samples. According to the modified Drude-Smith model (Eq. 7 in the main text), the DQ value should be between 0 and 1 . The $A \beta$ protein states were observed to be strongly related with the $\mathrm{DQ}$ values of $\sim 0$ for monomers, $\sim 0.64$ for an intermediate range of oligomers, and $\mathrm{DQ}=\sim 1$ for fibrils. Physically, in terms of free carrier dynamics, the DQ value implied the scattering event from 0 (free electron) to 1 (fully localization). The fibrillar state of the $\mathrm{A} \beta$ protein for $\mathrm{DQ} \sim 1$ resembled the well-known carrier-localized effect in the semiconductor nanowire. ${ }^{5}$ In Figure S5, the DQ values were almost discrete, regardless of the sample conditions and $A \beta$ protein concentrations. However, large standard deviations of the DQ values in the dried sample result from the phase shift of the terahertz pulse due to sample inhomogeneity. DQ evaluation for the $A \beta$ protein strongly associated with the Alzheimer's disease, can be very efficient in verifying the degree of fibrillization.

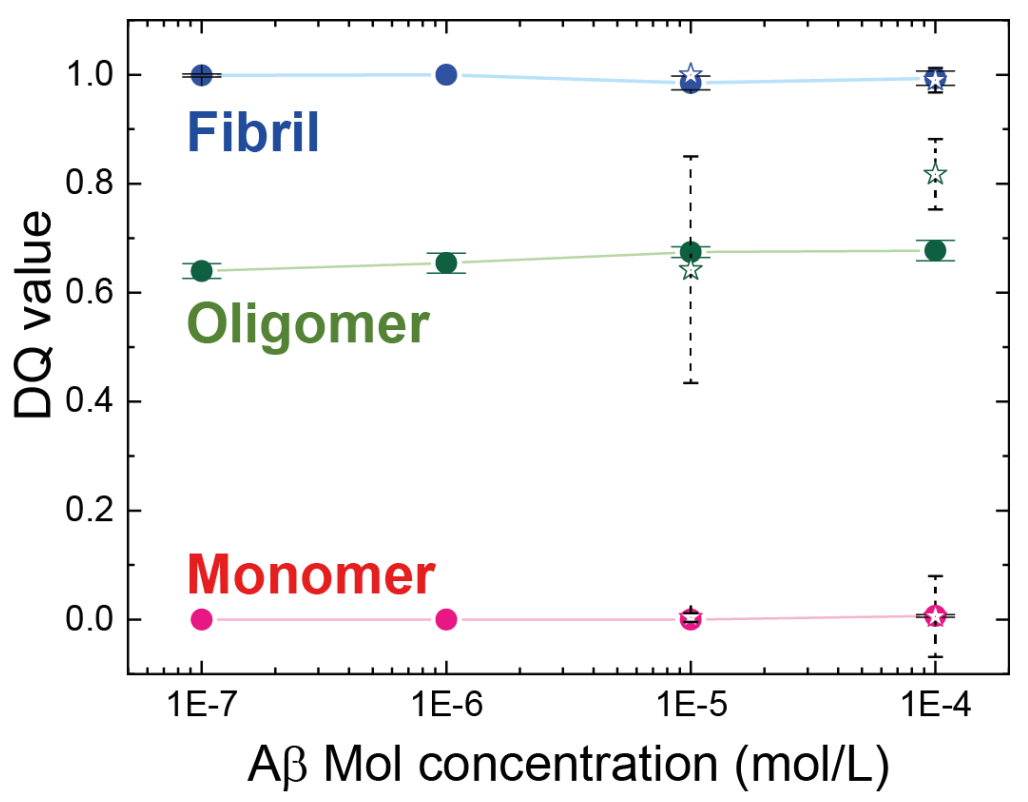

Figure S5. Discretized DQ values for the dried (star mark) and solution (solid circle) samples between 0 and 1 of the monomer, oligomer, and fibril $A \beta$, extracted from modified Drude-Smith model. The large standard deviation in the dry sample appeared to be irrevocable. 


\section{Note 7. A $\beta$ protein preparation and fibrillization step}

We purchased the synthetic peptide of $A \beta$ protein and dissolved it in a solvent to obtain appropriate molar concentrations, based on the following flowchart. For the aggregated and non-aggregated formations of proteins, the $A \beta$ protein was incubated with different buffer solutions at different temperatures.
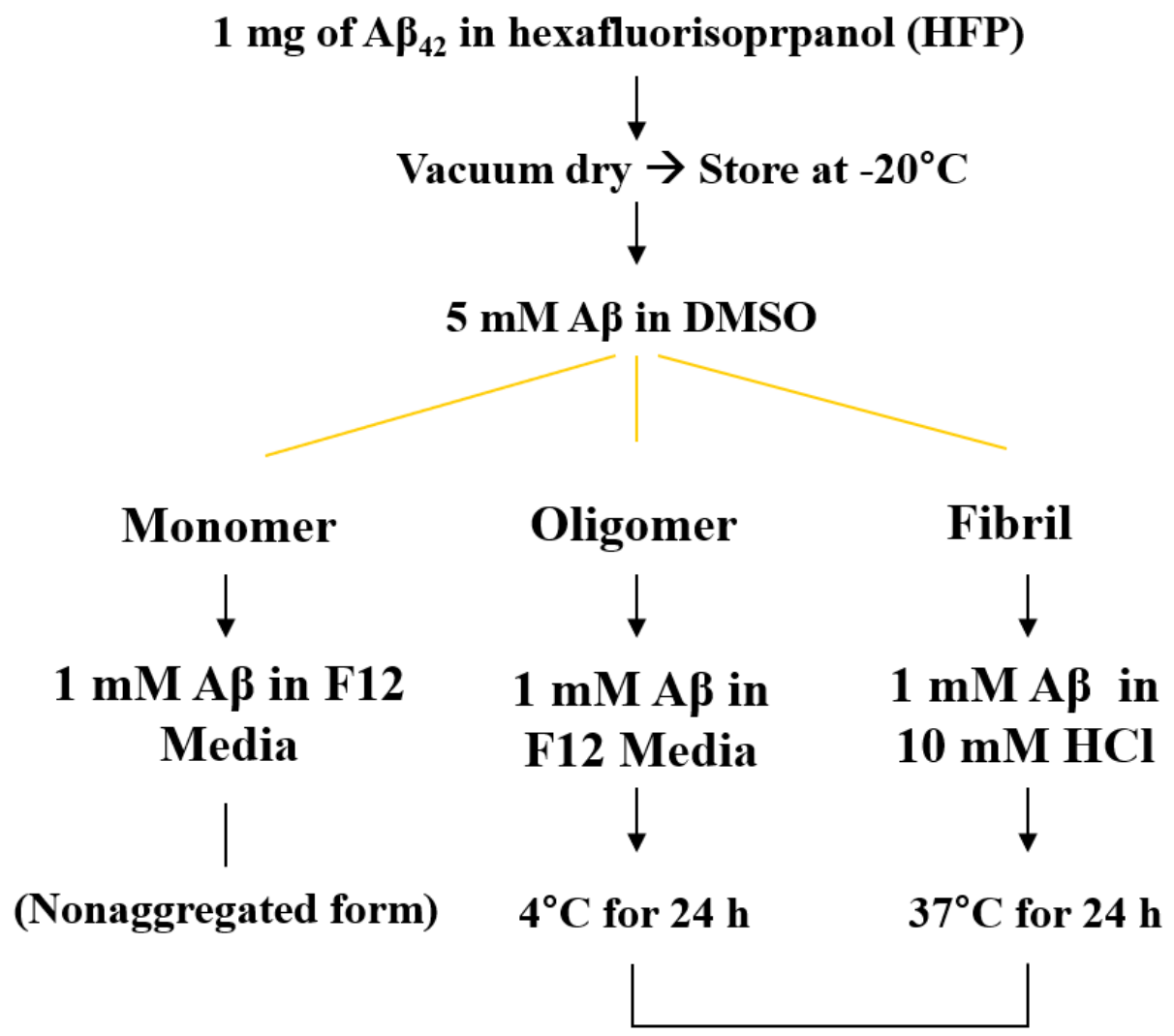

(Aggregated forms)

\section{REFERENCES}

1. Wilmink, G. J.; Ibey, B. L.; Rivest, B. D.; Grundt, J. E.; Roach, W. P.; Tongue, T. D.; Schulkin, B. J.; Laman, N.; Peralta, X. G.; Roth, C. C.; Cerna, C. Z. Development of a Compact Terahertz Time-Domain Spectrometer for the Measurement of the Optical Properties of Biological Tissues. J. Biomed. Opt. 2011, 16, 047006.

2. Glover, R. E.; Tinkham. M. Transmission of Superconducting Films at Millimeter-Microwave 
and Far Infrared Frequencies. Phys. Rev. 1956, 104, 844.

3. Kim, J.; Maeng, I.; Jung, J.; Song, H.; Son, J.-H.; Kim, K.; Lee, J.; Kim, C.-H.; Chae, G.; Jun, M.; Hwang, Y. K.; Lee, S. J.; Myoung, J.-M.; Choi, H. Terahertz Time-Domain Measurement of Non-Drude Conductivity in Silver. Appl. Phys. Lett. 2013, 102, 011109.

4. Niel, H.; Parente, M.; Baldi, A.; Gómez Rivas, J. Terahertz Time-Domain Spectroscopy and Near-Field Microscopy of Transparent Silver Nanowire Networks. Adv. Opt. Mat. 2020, 8, 1900790.

5. Ruhkopf, J.; Sawallich, S.; Nagel, M.; Otto, M.; Plachetka, U.; Kremers, T.; Schnakenberg, U.; Kataria, S.; Lemme, M. C. Role of Substrate Surface Morphology on the Performance of Graphene Inks for Flexible Electronics. ACS Appl. Electron. Mater. 2019, 1, 1909. 